\title{
Management strategies for gastrointestinal, erectile, bladder, and sudomotor dysfunction in patients with diabetes
}

\author{
P. Kempler ${ }^{1 *}$ G. Amarenco ${ }^{2}$ \\ R. Freeman ${ }^{3}$ S. Frontoni ${ }^{4}$ \\ M. Horowitz ${ }^{5}$ M. Stevens ${ }^{6}$ \\ P. Low ${ }^{7}$ R. Pop-Busui ${ }^{8}$ \\ A. A. Tahrani ${ }^{9,10}$ S. Tesfaye ${ }^{11}$ \\ T. Várkonyi ${ }^{12}$ D. Ziegler ${ }^{13,14}$ \\ P. Valensi ${ }^{15}$ on behalf of The \\ Toronto Consensus Panel on \\ Diabetic Neuropathy ${ }^{\dagger}$
}

${ }^{1}$ I Department of Medicine, Semmelweis University, Budapest, Hungary ${ }^{2}$ Service de Neuro-Urologie, Hôpital Tenon, Assistance Publique-Hôpitaux de Paris, Paris, France ${ }^{3}$ Beth Israel Deaconess Medical Center, Harvard Medical School, Boston, MA, USA ${ }^{4}$ Department of Internal Medicine, University of Tor Vergata, Rome, Italy ${ }^{5}$ Discipline of Medicine, University of Adelaide, Adelaide, SA, Australia ${ }^{6}$ Department of Medicine, University of Birmingham, Birmingham, UK ${ }^{7}$ Mayo Clinic, Rochester, MN, USA ${ }^{8}$ Division of Metabolism, Endocrinology and Diabetes, Brehm Center for Diabetes Research, University of Michigan, Ann Arbor, MI, USA ${ }^{9}$ Department of Diabetes and Endocrinology, Heart of England NHS Foundation Trust, Birmingham, UK

${ }^{10}$ School of Clinical and Experimental Medicine, University of Birmingham, Birmingham, UK ${ }^{11}$ Diabetes Research Unit, Sheffield Teaching Hospitals, Sheffield, UK ${ }^{12}$ First Department of Medicine, University of Szeged, Szeged, Hungary ${ }^{13}$ Institute for Clinical Diabetology, German Diabetes Center, Leibniz Center for Diabetes Research, Heinrich Heine University, Düsseldorf, Germany ${ }^{14}$ Department of Metabolic Diseases, University Hospital, Düsseldorf, Germany ${ }^{15}$ Service

d'Endocrinologie-Diabétologie-Nutrition, AP-HP, Hôpital Jean Verdier, Paris-Nord University, CRNH-IdF, Bondy, France

*Correspondence to: P. Kempler, I Department of Medicine, Semmelweis University, 1083 Budapest, Korányi Sándor u. 2/a, Hungary E-mail: kempet@bel1.sote.hu

†See Appendix for Members of The Toronto Consensus Panel on Diabetic Neuropathy.

Received: 12 April 2011

Accepted: 6 June 2011

\section{Summary}

There are substantial advances in understanding disordered gastrointestinal autonomic dysfunction in diabetes. It occurs frequently. The underlying pathogenesis is complex involving defects in multiple interacting cell types of the myenteric plexus as well. These defects may be irreversible or reversible. Gastrointestinal symptoms represent a major and generally underestimated source of morbidity for escalating health care costs in diabetes. Acute changes in glycaemia are both determinants and consequences of altered gastrointestinal motility. 35-90\% of diabetic men have moderate-to-severe erectile dysfunction (ED). ED shares common risk factors with CVD. Diagnosis is based on medical/sexual history, including validated questionnaires. Physical examination and laboratory testing must be tailored to patient's complaints and risk factors. Treatment is based on PDE5-inhibitors (PDE5I). Other explorations may be useful in patients who do not respond to PDE5-I. Patients at high cardiovascular risk should be stabilized by their cardiologists before sexual activity is considered or ED treatment is recommended.

Estimates on bladder dysfunction prevalence are $43-87 \%$ of type 1 and $25 \%$ of type 2 diabetic patients, respectively. Common symptoms include dysuria, frequency, urgency, nocturia and incomplete bladder emptying. Diagnosis should use validated questionnaire for lower urinary tract symptoms. The type of bladder dysfunction is readily characterized with complete urodynamic testing.

Sudomotor dysfunction is a cause of dry skin and is associated with foot ulcerations. Sudomotor function can be assessed by thermoregulatory sweat testing, quantitative sudomotor axon reflex test, sympathetic skin response, quantitative direct/indirect axon reflex testing and the indicator plaster. Copyright $\odot 2011$ John Wiley \& Sons, Ltd.

Keywords gastroparesis; gastrointestinal autonomic neuropathy; erectile dysfunction; sudomotor dysfunction; bladder dysfunction

Abbreviations AGE - advanced glycation end products; $\mathrm{BPH}$ - benign prostatic hyperplasia; CAN - cardiovascular autonomic neuropathy; cGMP cyclic guanosine monophosphate; CVD-cardiovascular disease; DPNdiabetic polyneuropathy; DBSQ - Diabetes Bowel Symptom Questionnaire; GIP-gastro inhibitory polypeptide; GLP-glucagon-like peptide; HRV-heart rate variability; ICC-interstitial cells of Cajal; LDF-laser Doppler flowmetry; LDPI-laser Doppler perfusion imaging; LSCI-laser speckle contrast imaging; LUTS-lower urinary tract symptoms; MBF-microvascular blood flow; NOS-nitric oxide synthase; PDE5-I-phosphodiesterase type 5 inhibitor; PGE1 - prostaglandin E1; PMN-polymorphonuclear leukocyte; PVR-postvoid residual volume; QDIRT-quantitative direct and indirect axon reflex testing; 
QoL-quality of life; QSART-quantitative sudomotor axon reflex test; RCT-randomized controlled trial; SFN-small fibre neuropathy; SSEP-somatosensoryevoked potentials; SSR-sympathetic skin response; TST - thermoregulatory sweat testin

\section{Introduction}

In this article, gastrointestinal, erectile, bladder and sudomotor dysfunction are reviewed. Disorders such as neuroarthropathy, hypoglycaemia unawareness, neuropathic oedema and pupillary abnormalities, which are in part due to autonomic dysfunction, are not covered in this article.

\section{Gastrointestinal autonomic neuropathy}

\section{Epidemiology}

Disordered gastrointestinal motility in diabetes may be associated with gastrointestinal symptoms, impaired oral drug absorption, poor glycaemic control, malnutrition and abnormal postprandial regulation of blood pressure. Reports on the prevalence of gastrointestinal symptoms and disordered motility in diabetes are inconsistent reflecting, partly, variations in methodology and the populations studied. Gastrointestinal symptoms impact negatively on health-related quality of life and women appear to be affected more frequently. The relationships among symptoms, disordered gastrointestinal motility and CAN are relatively weak.

Oesophageal transit, as measured using radionuclide techniques, is delayed in $\sim 50 \%$ of patients with longstanding diabetes, but does not correlate closely with delayed gastric emptying [1]. Other than acid reflux, oesophageal dysfunction can result in regurgitation, dysphagia and a propensity for pill-induced oesophageal erosions and structures.

Gastroparesis, defined as a delayed gastric emptying in the absence of an obstructive aetiology, is arguably the most important manifestation of gastrointestinal autonomic neuropathy affecting about $30-50 \%$ of outpatients with longstanding type 1 or type 2 diabetes $[1,2]$. The prevalence is highest when gastric emptying of both solids and nutrient-containing liquids is quantified [1,2]. Abnormally rapid gastric emptying was also described in diabetes [3]. Symptoms of gastroparesis usually relate to impaired gastric relaxation and 'hypersensitivity' to gastric distension. Only postprandial fullness appears to be a significant predictor of delayed gastric emptying of solids. Symptoms attributable to gastroparesis are reported in $5-12 \%$ of diabetic patients in the community, but higher rates are evident in patients evaluated in tertiary referral centres [4]. Symptoms seem to be more common in those with poor chronic glycaemic control, while acute hyperglycaemia has been shown to increase the perception of gastrointestinal sensations. Symptoms occur more frequently in patients with markers of psychological disorders. Symptom 'turnover' occurs frequently [2]. While patients with symptomatic gastroparesis utilize substantially more healthcare resources than diabetic patients without gastroparesis, moderate gastroparesis does not appear to be a rapidly progressive disease and is not associated with poor adverse prognosis [2].

Gastric emptying rate is of fundamental importance to postprandial glycaemia, accounting for at least a third of the variance of the initial rise, as well as the peak blood glucose response to carbohydratecontaining meals in healthy subjects, and patients with type 1 and type 2 diabetes. Gastric emptying rate is also a determinant of the incretin hormone responses which further affect postprandial glycaemia [5,6]. Even minor changes in the rate of carbohydrate delivery to the small intestine can have a substantial effect on glycaemia [6]. In insulin-treated patients nutrient delivery needs to be matched with the action of exogenous insulin and delayed gastric emptying has recently been documented as an important cause of otherwise unexplained hypoglycaemia - a phenomenon called 'gastric' hypoglycaemia [7]. In contrast, in type 2 diabetic patients not treated with insulin, a delayed carbohydrate absorption is often beneficial for glycaemic control.

Diabetes is also associated with a high prevalence of disordered small and large intestinal, and anorectal motility [8]. Either delayed or rapid small intestinal transit may be observed and may influence carbohydrate absorption. Diarrhoea is reported by up to $20 \%$ of patients and may reflect rapid or slow transit, frequently complicated by bacterial overgrowth. There is little information about colonic function in diabetes, but constipation has been reported in up to $60 \%$ of patients with longstanding diabetes [8]. An increased prevalence of faecal incontinence, particularly nocturnal, is related to reduced and unstable internal anal sphincter tone, impaired rectal compliance and sensation.

\section{Pathogenesis}

Gastrointestinal motor, sensory and secretory functions are modulated by the interaction of autonomic and enteric nervous systems with underlying rhythmicity generated by the so-called ICC located within the smooth muscle. The autonomic nervous system provides extrinsic innervation of gut function, via parasympathetic (vagal and pelvic) and sympathetic (mesenteric) nerves. Parasympathetic innervation is both excitatory (cholinergic) and inhibitory (non-adrenergic, non-cholinergic), whereas sympathetic input is generally inhibitory, with the exception of the lower oesophageal and anal sphincters. The pathogenesis of gastrointestinal 'autonomic neuropathy' is now recognized to be complex and multifactorial with 
defects in various interacting cell types and potentially, reversible, functional abnormalities, in addition to the more established roles of autonomic neuropathy and acute hyperglycaemia.

Data from the NIH-funded Gastroparesis Clinical Research Consortium have contributed significantly to knowledge of the role of cellular defects underlying disordered gut function, particularly the pathogenesis of gastroparesis. Documented changes include reduced numbers of ICC, deficiencies of inhibitory neurotransmission, reduced numbers of extrinsic autonomic neurons and smooth muscle [9]. Loss of dysfunction of ICC appears to be central to the pathogenesis of diabetic gastroparesis [10]. In animal models and humans with diabetic gastroparesis, a reduction in intraneuronal levels of nitric oxide has been observed.

Acute variations in glycaemia have a major impact on gut motor function in both healthy and diabetic subjects [5]. In type 1 patients, marked hyperglycaemia (16-20 mmol/L) slows gastric emptying of solids and nutrient-containing liquids substantially. Even within the physiological postprandial glycaemic range, the rate of gastric emptying is slower at a blood glucose of $8 \mathrm{mmol} / \mathrm{L}$ than $4 \mathrm{mmol} / \mathrm{L}$, in healthy subjects and patients with uncomplicated type 1 diabetes [11]. In contrast, insulininduced hypoglycaemia accelerates gastric emptying even in type 1 patients with gastroparesis. Hyperglycaemia also increases the perception of gastrointestinal symptoms in patients with diabetes [12]. Potential mediators of the effects of glycaemia on gut motility include impaired vagal function, altered prostaglandin synthesis, changes in nitric oxide mechanisms and both central and peripheral glucose-responsive neurons. The effects of variations in glycaemia in type 2 diabetes are less well documented.

\section{Diagnosis}

Studies of the autonomic neuropathy in diabetes, whether performed for clinical, epidemiological or research purposes, may potentially focus on gastrointestinal symptoms, QoL, gastrointestinal motility/transit, glycaemic control and/or postprandial blood pressure. A number of measurements are available to quantify gastrointestinal symptoms, including the DBSQ, which has been validated in a diabetic population. QoL measures have not focused specifically on the gastrointestinal tract.

Due to the poor predictive value of symptoms, objective measurements of gastric emptying are advocated for the diagnosis of gastroparesis. Evaluation of solid emptying is probably more sensitive than that of low-nutrient liquid or semi-solid meals. However, some patients will only exhibit delay to liquid meals. Gastric emptying can be affected acutely by many factors, including medications, smoking and blood glucose concentrations. Other causes of gastroparesis must be excluded. Medications that may influence gastric emptying should ideally be withdrawn for 48-72 $\mathrm{h}$ prior to the test, smoking should be avoided on the test day and the blood glucose concentration should be monitored and ideally be $>4$ and $<10 \mathrm{mmol} / \mathrm{L}$ during the test [11]. Failure to demonstrate delayed gastric emptying need not imply that symptoms are not due to 'diabetic gastropathy'.

Scintigraphy is still regarded as the 'gold standard' technique for measurement of gastric emptying. The intragastric distribution of a meal, which is frequently abnormal in diabetic patients, can also be evaluated. The level of standardization of the technique between centres is a major limitation, rectified to some extent by recent 'consensus' guidelines which recommend a low-fat, egg white meal labelled with $99 \mathrm{mTc}$ sulfur colloid and consumed with jam and toast as a sandwich, with a glass of water [2]. If carbohydrate is included in the meal the relationship between glycaemic response and gastric emptying rate can be evaluated [1]. The limitations of scintigraphy are exposure to a modest dose of radiation, the relative expense and the need for specialist centres.

Breath test results using non-radioactive ${ }^{13} \mathrm{C}$-acetate or -octanoic acid as a label are safe, inexpensive and correlate well with scintigraphy results $[2,13]$.

Ultrasonography is a non-invasive diagnostic method and two-dimensional ultrasound has been validated for measuring emptying of liquids and semi-solids. Three-dimensional ultrasound offers, however, a more comprehensive imaging of the total stomach. Magnetic resonance imaging has been used to measure gastric emptying and motility with excellent reproductivity, but its use is limited to research purposes. Surface electrogastrography should be considered currently as a research tool. A barium meal has a place in evaluating mucosal lesions or obstruction. Pressure measurements assessing motor function of the stomach, small intestine and colon correlate relatively well with scintigraphy results [13]. Manometry should be considered as a research technique to investigate gastric and intestinal motility. Tests of anorectal motor and sensory functions are well developed for clinical use.

\section{Management}

\section{Gastrointestinal symptoms}

The management of patients with symptomatic diabetic gastrointestinal autonomic neuropathy should focus on relief of gastrointestinal symptoms, improvement in nutritional status and optimization of glycaemic control. Patients with type 1 diabetes may benefit from insulin pump therapy.

Patients with disordered oesophageal motility associated with reflux should be managed conventionally, usually with proton pump inhibitors. Fluids should be consumed immediately after medications to minimize the possibility of pill-induced oesophagitis.

In patients with symptomatic gastroparesis, lowfat/fibre diets are frequently advocated, but their use is 
empirical. Prokinetic drugs, including metoclopramide, erythromycin and domperidone, are the mainstay of treatment. Their effect is due to the stimulation of acetylcholine release in the myenteric plexus to dopamine antagonist properties or by stimulating motilin receptors in the gut. The acceleration of gastric emptying by prokinetics is greater if baseline emptying is more delayed and their effect is attenuated during acute hyperglycaemia [14]. In a systematic analysis of clinical trials comparing various prokinetics, erythromycin appeared to be superior in accelerating gastric emptying and relieving symptoms [15], but its long-term efficacy is limited by tachyphylaxis due to down-regulation of motilin receptors, gastrointestinal adverse effects and, possibly, an increased risk of cardiac death. The adverse effects of the central nervous system are common and irreversible tardive dyskinesia is a rare complication with the use of metoclopramide. Domperidone is also effective at relieving symptoms and may now be regarded as the current 'first-line' agent, subsequent to restrictions in the availability of cisapride due to arrhythmias related to QT interval prolongation. Several drugs, including the motilin agonist, mitemcinal, ghrelin and ghrelin receptor agonists, 5HT4-receptor agonists and the muscarinic antagonist, acotiamide, are being currently investigated for their potential use [10]. Non-pharmacological treatments for diabetic gastroparesis are available. High-frequency gastric electrical stimulation with the Enterra device has been used widely, study results are controversial and have failed to provide conclusive evidence of benefit [16]. Benefits of surgical therapy for intractable gastroparesis remain uncertain. Uncontrolled observations support the benefit of pancreatic transplantation on gastric emptying.

The management of diarrhoea, constipation and faecal incontinence is still largely symptomatic once specific causes like coeliac disease and exocrine pancreatic insufficiency are excluded [17]. Bacterial overgrowth, implicated in up to $40 \%$ of diabetic patients with chronic diarrhoea, should be treated with antibiotics. Patients with accelerated transit or faecal incontinence may respond to loperamide, which slows small and large intestinal transit and increases internal anal sphincter tone [17]. In cases of severe diarrhoea somatostatin analogues may be effective.

\section{Glycaemic control}

The novel insights relating to the impact of gastric emptying on glycaemia have stimulated the development of dietary and pharmacological strategies to improve glycaemic control by modulating gastric emptying. The relationship of glycaemia to small intestinal carbohydrate delivery is now recognized to be nonlinear, as evidenced by the glycaemic response to intraduodenal infusion of glucose at rates within the normal range for gastric emptying in healthy and type 2 diabetic patients.
Non-pharmacological strategies include dietary modifications to slow gastric emptying by increasing dietary fibre and the use of fat or protein 'preloads' taken before a meal [18]. The rationale for the latter approach is to slow gastric emptying by stimulating small intestinal neurohumoral feedback mechanisms and the release of GIP and GLP-1 before the main meal. Slowing of gastric emptying may be the predominant mechanism by which exogenous GLP-1 and its analogues reduce postprandial glycaemia. The magnitude of slowing gastric emptying by exenatide is most marked when gastric emptying is more rapid and seems not to be attenuated by the presence of CAN [19].

Postprandial hypotension occurs frequently in diabetes (probably more commonly than orthostatic hypotension). The magnitude of the fall in blood pressure is related directly to the rate of gastric emptying when small intestinal carbohydrate delivery is more rapid, and the fall in blood pressure is greater. Dietary pharmacological strategies slowing gastric emptying and reducing small intestinal carbohydrate absorption are likely to prove effective in the management and there is evidence that the $\alpha$-glucosidase inhibitor, acarbose, may be useful. Delayed gastric emptying influences the delivery and absorption of orally administered drugs in the small intestine, resulting in later, or fluctuating maximal serum concentrations. This is particularly important if rapid onset drug action is required.

\section{Summary and recommendations}

During the past 25 years there has been a substantial redefinition of concepts relating to the prevalence, clinical significance, pathogenesis and management of 'gastrointestinal autonomic neuropathy' in diabetes. The pathogenesis is now recognized to be complex and heterogeneous. Gastrointestinal symptoms occur frequently in diabetes and affect QoL adversely. Diabetes is the most common cause of chronic gastroparesis affecting 30-50\% of patients with longstanding diabetes and the rate of gastric emptying is a major determinant of postprandial glycaemia. Management is frequently suboptimal and should focus on the relief of gastrointestinal symptoms as well as improvement in nutritional status and glycaemic control.

1. Inquire routinely about gastrointestinal symptoms (C).

2. Measure gastric emptying, by either scintigraphy or stable isotope breath test, in patients with suspected gastroparesis (B).

3. Initiate therapy with a prokinetic drug and optimize glycaemic control in patients with symptomatic gastroparesis (B).

4. Recognize that gastroparesis may impact adversely on glycaemic control, particularly in insulin-treated patients (C). 


\section{Erectile dysfunction}

\section{Definition}

Erectile dysfunction is defined as persistent inability to achieve or maintain an erection sufficient to permit satisfactory sexual intercourse. Normal penile erection is the result of a hemodynamic process and is dependent on corporal smooth muscle relaxation mediated by parasympathetic neurotransmission, nitric oxide, electrophysiologic events and other regulatory factors.

\section{Epidemiology}

Erectile dysfunction prevalence among diabetic men varies from 35 to $90 \%$ depending mainly on the population studied and various methods applied. In a nationally representative study from managed care claims in the US database, diabetes prevalence rates were $20.0 \%$ in men with erectile dysfunction and $7.5 \%$ in men without erectile dysfunction [20]. The Cologne Male Survey noted a fourfold increase in erectile dysfunction in men with diabetes compared to the general population. The presence of diabetes was associated with an odds ratio for erectile dysfunction by 3.95 (2.98-5.23). Erectile dysfunction prevalence in the younger age groups (40-60 years) with diabetes was as high as in the older groups of non-diabetic subjects (60-80 years). In another survey, erectile dysfunction frequency among diabetic men aged 45-49 years was similar to that in non-diabetic men aged over 70 years [21]. In the EURODIAB IDDM Complications Study [22], $16 \%$ of patients (range: $2-35 \%$ ) had problems with intercourse, $16 \%$ (range: $2-85 \%$ ) had problems obtaining an erection, $18 \%$ (range: $2-83 \%$ ) had problems sustaining an erection, while 35\% (range: $23-84 \%$ ) had reported not to have erections at night or in the morning. Among 713 men with type 1 diabetes participating in the Diabetes Control and Complication Trial/Epidemiology of Diabetes Interventions and Complications Study (DCCT/EDIC), erectile dysfunction was present in 34\%, orgasmic dysfunction in 20\% and decreased libido in 55\% at year 10 of Epidemiology of Diabetes Interventions and Complications Study follow-up and after a mean diabetes duration of the cohort of 22.1 years [23]. The crude incidence rate of erectile dysfunction in the Massachusetts Male Aging Study was 26 cases/1000 man-years in 847 men aged 40-69 without erectile dysfunction at baseline, who were followed for an average of 8.8 years [24]. The age-adjusted erectile dysfunction risk was higher for men with lower education, diabetes, heart disease and hypertension. Erectile dysfunction incidence rate in diabetic men was increased twofold, with 50 cases/1000 man-years. In a study from Italy that included 9868 men with diabetes, $45.5 \%$ of those aged $>59$ years reported erectile dysfunction. Risk factors and clinical correlates included the following (odds ratio [95\% CI]): autonomic neuropathy (5.0 [3.9-6.4]), diabetic foot (4.0 [2.9-5.5]), peripheral neuropathy (3.3 [2.9-3.8]), peripheral arterial disease (2.8 [2.4-3.3]), nephropathy (2.3 [1.9-2.8]), poor glycaemic control (2.3 [2.0-2.6]), retinopathy $(2.2$ [2.0-2.4]), hypertension (2.1 [1.6-2.9]) and diabetes duration (2.0 [1.8-2.2]) [25]. However, even when neuropathic complications are present, psychiatric illnesses may be important contributors to erectile dysfunction in diabetic men. The aetiology of psychogenic erectile dysfunction includes anxiety, depression, sexual phobias and stress. Thus, psychogenic components must not be overlooked. The key differences between psychogenic and organic erectile dysfunction are shown in Table 1.

Erectile dysfunction is a well-recognized index of cardiovascular risk and an independent predictor of coronary artery disease. Prospective studies have shown that erectile dysfunction predicts the development of CVD [26] and CVD mortality [27]. The risk of CVD associated with erectile dysfunction is in the range of risk associated with traditional CVD risk factors $[27,28]$, such as current smoking, hypertension or family history of myocardial infarction, but erectile dysfunction does not improve the prediction of who will and will not develop CVD beyond that offered by traditional risk factors. New onset or progressive decline in erectile dysfunction should be considered as an alarming marker of threatening ischaemic heart disease even at asymptomatic stages.

Erectile dysfunction correlates significantly with the severity of CAN among type 1 diabetic patients. Although erectile dysfunction is associated with autonomic neuropathy, erectile dysfunction prevalence in patients with CAN and the prevalence of CAN among patients with erectile dysfunction have not been analysed in large epidemiological studies.

Longitudinal studies are essential to assess the natural course of erectile dysfunction. In a 1-year longitudinal study [28], the health-related quality of life and erectile dysfunction severity were compared in men with and without the history of diabetes. Diabetic men with a newly started erectile dysfunction treatment had favourable results at 6 months, but the response to therapy was not durable. In the study [29] with the highest number of participants' $(n=1456)$ deterioration in QoL just as worsening of depressive symptoms preceded the onset of erectile dysfunction.

\section{Pathogenesis}

The prominent role of neuropathy in the pathogenesis of erectile dysfunction is mainly explained by decreased smooth muscle relaxation of the corpus cavernosum and insufficient NOS function. The impaired sensation of the glans and abnormal motor function of muscles participating in erection also contribute to erectile dysfunction development among patients with neuropathy. Erectile dysfunction pathogenesis in diabetes is multifactorial, related to neuropathy, accelerated atherosclerosis and alterations in the corporal erectile tissue, including smooth muscle degeneration, abnormal 
Table 1. The classification of erectile dysfunction: psychogenic or organic?

\begin{tabular}{|c|c|c|}
\hline Aetiology & Psychogenic & Organic \\
\hline Onset & Sudden onset & Gradual onset \\
\hline Initiation & Complete immediate loss & Incremental progression \\
\hline Morning erection & Morning erections present & Lack of morning erections \\
\hline $\begin{array}{l}\text { Presence or absence under certain } \\
\text { circumstances }\end{array}$ & Varies with partner and circumstance & $\begin{array}{l}\text { Lack of erections under most sexually } \\
\text { stimulating circumstances }\end{array}$ \\
\hline
\end{tabular}

collagen deposition and endothelial cell dysfunction. AGE have been shown to quench NO and to be elevated in human diabetic penile tissue.

\section{Diagnosis}

Good clinical history and physical examination are the basis of assessment. It is important to establish the nature of the erectile problem and to distinguish it from other forms of sexual difficulty such as penile curvature or premature ejaculation. An interview with the partner is advisable and will confirm the problem but may also reveal other causes of difficulties, e.g. vaginal dryness. The relative importance of psychological and organic factors may be determined from the history. Drugs associated with erectile dysfunction include tranquillizers, antidepressants (tricyclics, selective serotonin reuptake inhibitors) and antihypertensives (ß-blockers, vasodilators, central sympathomimetics, ganglion blockers, diuretics, ACE inhibitors). Key diagnostic procedures of erectile dysfunction include comprehensive history (sexual, medical, drug use, risk factor assessment and psychosocial factors) [30]. Routine laboratory tests should include $\mathrm{HbA1c}$, fasting blood glucose and lipid profile. The necessity of total testosterone (if available: bio-available or free testosterone instead of total) measurement is currently discussed, but it is clearly necessary in patients who do not respond to PDE5-Is. The use of validated questionnaires, such as the International Index of Erectile Function and the Sexual Encounter Profile, is the most appropriate method to characterize frequency and severity of erectile dysfunction symptoms. Other explorations that may be useful in patients who do not respond to PDE5Is include evaluations of nocturnal penile tumescence, penile Doppler ultrasound, bulbo-cavernosus reflex, dorsal sensory nerve conduction of the penis, amplitude and latency of penile SSR, pudendal nerve SSEP, assessment of the effect of PGE1 on erection, psychological evaluation and urodynamic studies. Cardiac risk factors should be evaluated and managed in all erectile dysfunction patients. Three risk categories were established at the first and second Princeton Consensus Conferences [31]. After categorization of patients, an algorithm should be followed according to individualized risk stratification. As a first step sexual function assessment and initial cardiovascular evaluation are recommended. The second step is to initiate or resume sexual activity or indicate treatment of erectile dysfunction in low-risk patients. Patients at high risk should be stabilized by cardiological treatment before sexual activity is considered or erectile dysfunction treatment is recommended. The third step involves seeking all further risk factors of atherosclerosis in the presence of erectile dysfunction.

\section{Management}

A stepwise therapeutic approach for erectile dysfunction recently suggested by the European Association of Urology is shown in Figure 1 [32]. Even if the cause is organic, almost all men with erectile dysfunction are affected psychologically. Sexual counselling is an important aspect of treatment and should also involve the partner. The initial management should advise the patient to reduce possible risk factors and optimize glycaemic control. However, no studies have shown that improvement in glycaemic control will exert a favourable effect on erectile dysfunction. Lifestyle modification is associated with improvement in erectile dysfunction in obese men.

Most men consider oral agents as the first treatment choice. First-line oral therapy includes PDE5-Is (sildenafil, vardenafil and tadalafil) [32,33]. The mode of action of PDE5-Is is delaying of cGMP degradation. Increased cGMP level leads to decreased intracellular calcium levels, thereby producing smooth muscle relaxation in corpus cavernosum and enhancement in blood flow resulting in erection during sexual stimulus.

Meta-analysis of trials comparing treatment with various PDE5-Is with placebo in diabetic patients demonstrates the improvement of erectile dysfunction in diabetic men without reporting more frequent cardiovascular adverse events. The use of PDE5-Is together with oral nitrates is contraindicated. The efficacy of PDE5-Is is independent of the duration of diabetes, glycaemic control and microvascular complications. In diabetic patients the proportion of subjects reporting improved erection following treatment with any PDE5-I is lower compared to general population.

Daily use of 2.5 and $5 \mathrm{mg}$ tadalafil for 12 weeks was well tolerated and significantly improved erectile dysfunction in diabetic men [34]. However, these studies lacked an on-demand treatment arm. Daily use of tadalafil provides an alternative to on-demand dosing for couples who prefer spontaneous rather than scheduled sexual activity [32].

Treatment options for patients who do not respond to PDE5-Is or for whom PDE5-Is are contraindicated include intracavernous injections, intraurethral 


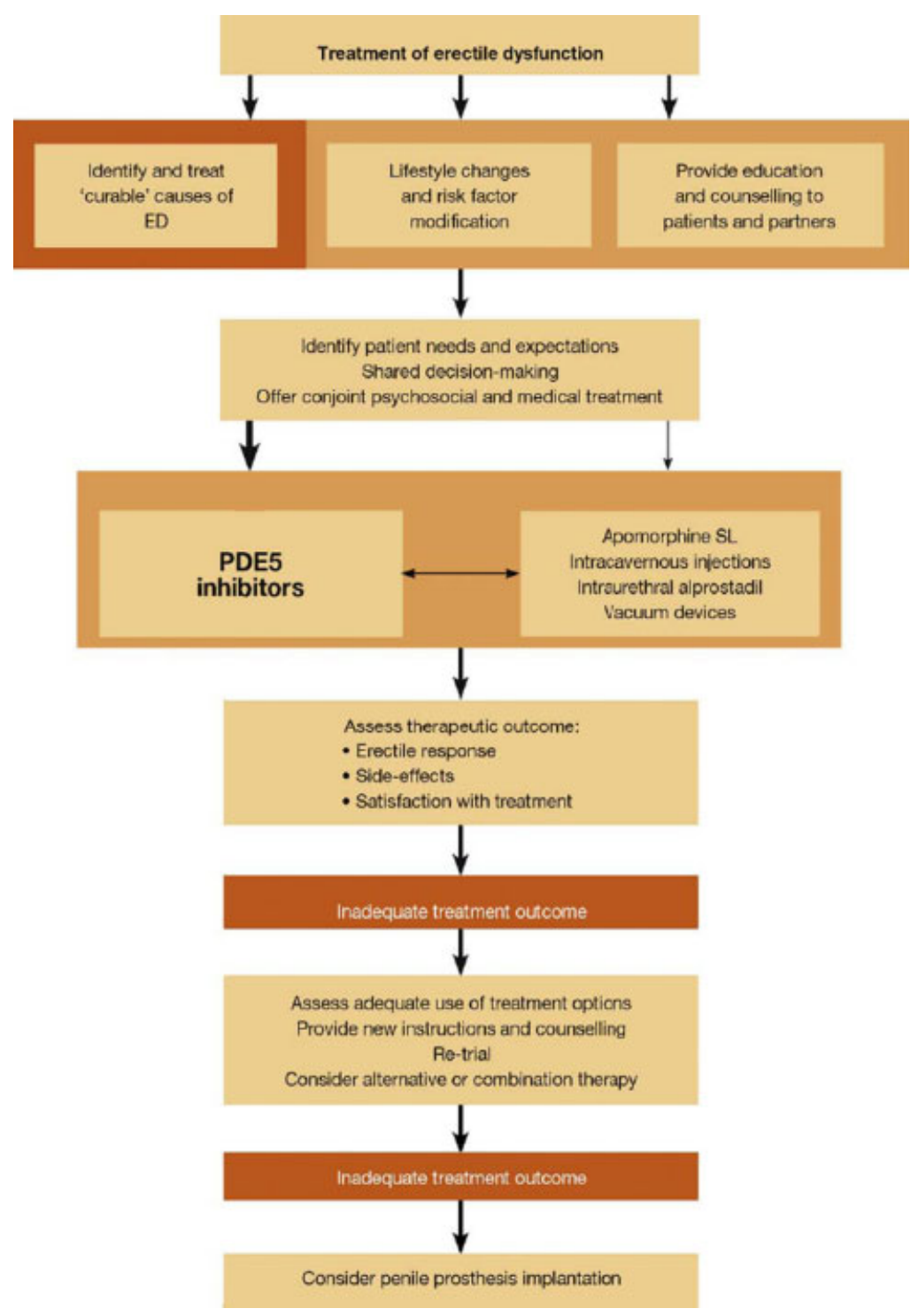

Figure 1. Treatment algorithm for erectile dysfunction in diabetic men (reproduced from Ref. [32])

alprostadil, vacuum constriction devices or penile prosthesis implantation [32].

\section{Summary and recommendations}

Moderate-to-severe erectile dysfunction is present in 5-20\% diabetic men. Erectile dysfunction shares common risk factors with CVD. Diagnosis is based on medical and sexual history, including validated questionnaires. Physical examination and laboratory testing must be tailored to the patient's complaints and risk factors. Treatment is based on PDE5-Is. PDE5-Is have high efficacy and safety rates in patients with diabetes.

1. Initiate therapy with a PDE5-I in men who seek treatment for erectile dysfunction, provided there is no contraindication for PDE5-I use [35] (A).

2. The choice of one specific PDE5-I should be based on the individual preferences of men with erectile dysfunction, including ease of use, cost of medication and adverse effects profile [35] (C).

3. Other choices may be useful in patients who do not respond to PDE5-Is (B).

4. Routine use of hormonal blood tests or hormonal treatment in the management of patients with erectile dysfunctions is not recommended [35] (E).

5. Patients at high cardiovascular risk should be stabilized by cardiological treatment before the sexual activity is considered or erectile dysfunction treatment is recommended (A).

\section{Bladder dysfunction}

Diabetes mellitus is associated with an earlier onset and increased severity of urologic diseases, specifically bladder disorders. Paralysis of the detrusor muscle, impairment of bladder sensation, alteration of urothelial receptors and urothelial signalling mechanisms are the main causes 
of urologic dysfunctions [36]. Diabetic cystopathy has a significant impact on QoL.

\section{Epidemiology}

Longstanding diabetes causes paralysis of the detrusor muscle leading to voiding difficulties, principally weak stream, PVR and impairment of bladder sensation.

Estimates on the prevalence of bladder dysfunction are $43-87 \%$ of type 1 diabetic patients and $25 \%$ of type 2 diabetic patients. The correlation between diabetic cystopathy and peripheral neuropathy ranged from 75 to $100 \%$ [37].

Diabetes duration, peripheral neuropathy and retinopathy are significantly associated with severe incontinence. In women, urinary incontinence is estimated to affect nearly $50 \%$ of middle aged and older women, leading to significant distress, limitations in daily functioning and poorer QoL. Diabetes has been identified as an important independent risk factor for incontinence in women in several large observational studies, including the Nurses' Health Study, and is associated with $30-100 \%$ increased risk. Women with diabetes are more likely to experience severe and symptomatic urinary incontinence. Type of incontinence is generally urge incontinence, an involuntary loss of urine with a feeling of urgency. Diabetic women treated with insulin are at considerably higher risk of urge incontinence than those treated with oral medications or diet [38].

In men, LUTS are common and often attributed to BPH. Among men with $\mathrm{BPH}$, diabetes is associated with more LUTS symptoms compared with non-diabetic men [39]. The effect of diabetes on the development or presence of LUTS has been studied more in patients with type 1 diabetes and is less clear in type 2 diabetes.

In the Diabetes Control and Complication Trial/ Epidemiology of Diabetes Interventions and Complications Study cohort, moderate-to-severe LUTS was reported in nearly $20 \%$ of men at year 10 of Epidemiology of Diabetes Interventions and Complications Study follow-up, after a mean diabetes duration of 22.1 years [40]. In the same cohort, among 550 women with type 1 diabetes who completed a self-administered questionnaire on incontinence, 38\% reported any incontinence and $17 \%$ reported weekly or greater incontinence [41]. Advancing age, increased weight and previous urinary tract infection were associated with higher risk of incontinence.

Straining, intermittency, postvoid dribbling and weak stream may be secondary to urethral obstruction from BPH. However, among men with diabetes, similar symptoms may also result from bladder dysfunction due to denervation and poor detrusor contractility

\section{Pathophysiology}

Bladder complications in diabetes can be due to an alteration in the detrusor smooth muscle, neuronal dysfunction and urothelial dysfunction. Responsiveness of diabetic bladder strips to externally applied muscarinic agonists has been reported as increased, decreased or described as little or no change in responsiveness.

The bladder urothelium is not a passive barrier only, playing an important role as a sensor, controlling bladder function. The effects of diabetes on urothelial receptors and urothelial signalling mechanisms have not been extensively studied, but it has been well established that in the streptozotocin-induced diabetic rat model, there are progressive increases in total bladder tissue with hypertrophy of the bladder wall and dilation of the bladder. The urothelial release of prostaglandins, which sensitize sensory nerves and bladder smooth muscle, may be increased in diabetes and contribute to some of the bladder abnormalities (e.g. detrusor overactivity) observed in diabetic bladder [42].

\section{Clinical evaluation}

Common symptoms include dysuria, frequency, urgency, nocturia and incomplete bladder emptying. Other symptoms include infrequent voiding, poor stream, hesitancy in initiating micturition, recurrent cystitis, stress and urgency urinary incontinence.

Urge incontinence, defined as involuntary loss of urine with feeling of urgency, is very frequent in women with DM, while there was no increased risk of stress incontinence, defined as involuntary loss of urine with physical activity.

Evaluation of the diabetic patients should include specific questions about urinary symptoms, ideally using a validated questionnaire for incontinence and LUTS. QoL evaluation by means of specific questionnaires is also very important. Assessment of perineal sensation, sphincter tone and the bulbo-cavernosus reflex may identify peripheral neuropathy consistent with diabetes. Complete urogynaecologic examination is needed to exclude pelvic organ prolapse or other pelvic disorders.

\section{Diagnosis}

Since diabetic patients are at increased risk of bacterial cystitis, microscopic urinalysis and culture are essential in assessing patients complaining of LUTS. The urothelium in diabetes is susceptible to infections particularly by Escherichia coli. Alterations in PMN function in highglucose state may contribute to an increased risk of urinary tract infection.

The diagnosis of diabetic neurogenic bladder and the exact type of dysfunction is made most readily with complete urodynamic testing. This may include cystometry, uroflow, simultaneous pressure/flow studies, sphincter electromyography and urethral pressure profilometry or evaluation of leak-point pressures. In addition, electrophysiologic testing or other assessments may be useful for the assessment of peripheral neuropathy. 
Detailed assessment of bladder function with urodynamic studies is indicated if initial management is unsuccessful or there is doubt about the diagnosis [38]. Measurement of peak urinary flow rate and PVR should be considered in diabetic patients with LUTS. PVR should ideally be measured using portable ultrasound, as invasive catheterization carries a potential risk of infection [43]. Women with type 2 diabetes have lower maximal flow rates compared to controls, being most severe in the presence of peripheral neuropathy [44].

Urodynamic findings include impaired bladder sensation, increased cystometric capacity, decreased detrusor contractility and increased PVR.

Diabetic patients who also had gastroparesis had delayed first sensation, increased capacity and increased PVR. Cystometric examination may show detrusor areflexia, but detrusor instability is also frequently found. Detrusor overactivity is the most common urodynamic observation (48\%) followed by impaired detrusor contractility (30\%), while $15 \%$ had poor compliance [45]. The exact mechanism of the detrusor overactivity in patients with diabetic cystopathy is unclear. Changes in bladder functions were observed as early as within 1 year from the diagnosis of diabetes.

In diabetic children, urinary flow disturbance was considered as an early sign of autonomic neuropathy [46]. Children with diabetes had a significantly increased voiding volume, increased average urinary flow and increased delay until the first sensation of the need to void, compared with healthy children. These abnormal urodynamic features are consistent with sensory nerve dysfunction. Time to maximum flow is longer in diabetic subjects as compared with controls and the acceleration (the ratio of maximum flow and the time to maximum flow) of diabetic patients is significantly lower. This decreased acceleration of detrusor muscle contraction may be interpreted as an early sign of autonomic neuropathy in diabetic children.

Perineal electrophysiological testing is altered in diabetic patients. Electromyography demonstrates peripheral neuropathy in perineal muscles (urethral striated sphincter, bulbo-cavernosus muscles). Bulbo-cavernosus reflexes are altered with an increase in sacral latencies. SSEP of tibial and pudendal nerves and motor-evoked potentials after transcranial magnetic stimulation are delayed in diabetes [47]. Abnormally prolonged tibial SSEP are statistically correlated with lower urinary tract dysfunction. On the contrary, abnormality of pudendal SSEP is not correlated to cystopathy.

Bladder dysfunction has not been assessed up to now in epidemiological and longitudinal studies or in RCTs.

\section{Summary and recommendations}

1. Diabetic cystopathy occurs in up to $80 \%$ of type 1 diabetic patients [48] (Class III).

2. Urinary incontinence is strongly associated with type 1 , but not with type 2 diabetes (Class II).
3. Patients with diabetic cystopathy have impaired detrusor contractions and increased PVR (Class II).

4. Overactive bladder is not uncommon in diabetes, presumably reflecting both central and peripheral mechanisms (Class III).

- PVR and urine dipstick (optional culture) should be performed yearly in all patients with insulindependent diabetes (C) [46].

- Treatment of choice for acontractile bladder remains intermittent catheterization (B/C) [46].

\section{Sudomotor dysfunction}

\section{Epidemiology}

Sudomotor function has not yet been assessed in epidemiological and longitudinal studies or in RCT's

\section{Pathogenesis}

Sweat glands are innervated by sudomotor, postganglionic, thin, unmyelinated cholinergic sympathetic Cfibres. C-fibres also contribute to MBF regulation. C-fibre dysfunction can occur early in the course of DPN.

\section{Clinical manifestations}

Sudomotor dysfunction is associated with dry skin and itching and may impair QoL and is associated foot ulceration in diabetes [49]. In addition, changes in cutaneous MBF can be associated with adverse changes in skin quality and structure, foot ulceration and oedema.

\section{Diagnosis}

Several methods have been developed to assess sudomotor function with variable degree of complexity and accuracy including TST, QSART, SSR, QDIRT, silicone impressions and the indicator plaster [50].

TST evaluates the integrity of central and peripheral sympathetic sudomotor pathways [51]. The core body temperature is raised to $38^{\circ} \mathrm{C}$ in a chamber controlled for humidity and a maximal sweat response recruited which is detected by a change in the indicator dye colour. Data are expressed as TST\% (anterior body anhidrosis area/anatomic figure area, $\times 100$ ). TST cannot differentiate pre- from postganglionic lesions, is time consuming, requires special equipment, facilities and preparation [49,51]. Sensitivity is $91-96 \%$ in patients with distal SFN (Class III) (C) [52]. Data specific to diabetes are lacking.

The indicator plaster represents a rapid, simple method based on the colour change of a cobalt II compound from blue to pink, after $10 \mathrm{~min}$ exposure to dermal foot perspiration at the plantar foot regions 
Table 2. Sensitivity and specificity of the indicator plaster in detecting peripheral neuropathy and autonomic neuropathy

\begin{tabular}{lcc}
\hline \multirow{2}{*}{ Authors } & \multicolumn{2}{c}{ Indicator plaster } \\
\cline { 2 - 3 } & Sensitivity (\%) & Specificity (\%) \\
\hline Peripheral neuropathy & & \\
Liatis et al. (A) & 86 & 67 \\
Spallone et al. (B) & 85 & 31 \\
Papanas et al. (C) & 94 & 70 \\
Papanas et al. (D) & 95 & 68 \\
Quattrini et al. (E) & 85 & 45 \\
Autonomic neuropathy & & \\
Liatis et al. (A) & 59 & 46 \\
Spallone et al. (B) & 82 & 27 \\
\hline
\end{tabular}

Reference are shown as online addendum (Supporting information).

[53]. Indicator plaster response correlated [54] with neuropathy disability score $(p<0.001)$, neuropathic symptom score $(p=0.03)$, cold detection threshold ( $p=$ 0.003), heat-as-pain perception threshold $(p<0.043)$ and deep-breathing $\operatorname{HRV}(p<0.00)$. Intra-epidermal nerve fibre density compared with age- and sex-matched control subjects was significantly reduced in patients with patchy/absent response $(p=0.02)$. The authors concluded that the indicator plaster may serve as a simple indicator for screening patients with diabetic neuropathy (C). Indicator plaster results correlate well with the presence of peripheral sensorimotor neuropathy and to lesser degree with indications of CAN (Table 2, references are shown in Supporting information). Although indicator plaster specificity was lower in most studies, it was comparable with other established methods (Class II and III); nonetheless, reference methods were different in the various studies.

QSART evaluates postganglionic sudomotor function [52]. Acetylcholine is iontophoresed and sweat production measured as an increase in humidity through a hygrometer [51]. Results are analysed using area under the curve, maximal sweat production and sweat onset latency. QSART is unable to detect preganglionic lesions, requires specialized equipment and is expensive [51]. Sensitivity is 54-90\% in patients with SFN (Class III) [52]. Combining QSART with TST improves the sensitivity to $98 \%$ and helps to localize the lesion [52]. In patients with painful diabetic neuropathy, QSART has poor sensitivity (58\%) and correlates with pain severity (Class III) [55].

SSR, surrogate measure of sudomotor function, measures electrodermal activity [51]. Responses may be absent in $>50$-year individuals and there is no clear definition of abnormal response [51]. A sensitivity of $87.5 \%$, a specificity of $88.2 \%$ and a negative predictive value of $93.7 \%$ to diagnose/exclude diabetic autonomic neuropathy (Class III) is reported [56]. SSR sensitivity in detecting diabetic (peripheral) neuropathy is $31-76 \%$ (Class III).

Silicone impressions use acetylcholine iontophoresis to stimulate sweating [51]. QDIRT has been validated against silicone impressions and QSART [57]. QDIRT measures the postganglionic sudomotor function [51] utilizing an indicator dye (alizarin red mixture) and sweat droplets are quantified [51]. QDIRT is very sensitive to temperature, humidity, hydration status and caffeine intake [51]. QDIRT results highly correlate with silicone images [58].

\section{Microvascular function assessment}

The skin offers an accessible organ to assess MBF and endothelial function, which correlate with systemic measurements of endothelial function and myocardial microcirculation [57]. Several methods are available to assess skin MBF [59]. Laser Doppler allows the determination of blood flow under baseal conditions or following physical (e.g. heating) or pharmacological (e.g. acetylcholine and/or sodium nitroprusside) stimulation, allowing the differentiation between endothelial-dependent and -independent responses [57]. Furthermore, laser Doppler allows the measurement of nerve axon reflexrelated vasodilation following acetylcholine iontophoresis which is the result of C-fibre stimulation. Laser Doppler techniques include LDF, LDPI and LSCI [57].

Single-point LDF measures flow within a small volume of the dermis $\left(1 \mathrm{~mm}^{3}\right)$, while LDPI scans across a larger area of skin [57]. In LSCI, speckles are formed by the backscatter of laser light and moving particles cause fluctuations in the speckle pattern which is related to the speed of the illuminated particles (such as blood cells). LDF and LPDI have been utilized in DPN [60], but data with LSCI are very limited.

LDF has a moderate sensitivity and specificity (75 and $69 \%$, respectively) when compared with SSR in patients with diabetic (peripheral) neuropathy (Class III) [61]. LDPI has a better performance (sensitivity 92\% and specificity 93\%) compared to nerve conduction studies in diabetic (peripheral) neuropathy (Class III) [62]. In assessing autonomic neuropathy, LDF measurements showed moderate correlations with CAN parameters.

Each of the above-mentioned laser Doppler techniques has its advantages/disadvantages. LDF has unacceptably high variation with the mean day-to-day coefficient of variation in excess of $30 \%$ compared to $6.4-12.1 \%$ with LDPI and 8\% with LSCI [60]. The theoretical model linking the outcome measurement to perfusion is well established and accepted with regard to LDPI but not for LSCI.

\section{Recommendations}

1. All methods mentioned above are appropriate for sudomotor function testing in diabetic patients, although some need further validation (Level C).

2. The indicator plaster may serve a simple method for screening of diabetic (peripheral) neuropathy (Level C).

3. SSR and QSART performance is better when detecting autonomic neuropathy compared to diabetic (peripheral) neuropathy (Level C). 
4. LDPI is a reproducible, sensitive and specific tool to diagnose diabetic (peripheral) neuropathy (Level C).

5. There is a need to standardize methods when using laser Doppler in clinical studies (Level C).

6. Studies validating the use and reproducibility of LSCI in diabetic (peripheral) neuropathy are needed (Level C).

7. Prospective studies are needed to assess the prognostic value of these tests (Level C).

\section{Supporting information}

Supporting information may be found in the online version of this article.

\section{Conflict of interest}

The authors have no conflicts of interest.

\section{Appendix}

The Toronto Consensus Panel on Diabetic Neuropathy James W Albers, MD, PhD, University of Michigan, Ann Arbor, MI, USA

Gérard Amarenco, MD, Service de Rééducation Neurologique et d'Explorations Périnéales, Hôpital Rothschild, AP-HP, Paris, France

Henning Anderson, MD, Department of Neurology, Aarhus University Hospital, Aarhus, Denmark

Joe Arezzo, PhD, Albert Einstein College of Medicine, New York, NY, USA

Misha-Miroslav Backonja, MD, Department of Neurology, University of Madison-Wisconsin, Madison, WI, USA

Luciano Bernardi, MD, Clinica Medica 1, Universita' di Pavia, Pavia, Italy

Geert-Jan Biessels, MD, Department of Neurology, Rudolf Magnus Institute, Utrecht, The Netherlands

Andrew J. M. Boulton, MD, Department of Medicine, University of Manchester, Manchester, UK

Vera Bril, MD, Department of Neurology, University of Toronto, Toronto, ON, Canada

Norman Cameron, PhD, University of Aberdeen, Aberdeen, UK

Mary Cotter, PhD, University of Aberdeen, Aberdeen, UK Peter J. Dyck, MD, Department of Neurology, Mayo Clinic, Rochester, MN, USA

John England, MD, Department of Neurology at Louisiana State University Health Sciences Center, New Orleans, LA, USA

Eva Feldman, MD, PhD, Department of Neurology, University of Michigan, Ann Arbor, MI, USA

Roy Freeman, MD, Beth Israel Deaconess Medical Center, Harvard Medical School, Boston, MA, USA

Simona Frontoni, MD, Department of Internal Medicine, University of Tor Vergata, Rome, Italy
Jannik Hilsted, MD, Copenhagen University Hospital, Copenhagen, Denmark

Michael Horowitz, $\mathrm{MD}, \mathrm{PhD}$, Department of Medicine, University of Adelaide, Adelaide, SA, Australia

Peter Kempler, MD, PhD, I Department of Medicine, Semmelweis University, Budapest, Hungary

Giuseppe Lauria, MD, Neuromuscular Diseases Unit, "Carlo Besta" Neurological Institute, Milan, Italy

Philip Low, MD, Department of Neurology, Mayo Clinic, Rochester, MN, USA

Rayaz Malik, MD, Division of Cardiovascular Medicine, University of Manchester, Manchester, UK

Peter C. O’Brien, PhD, Mayo Clinic, College of Medicine, Rochester, MN, USA

Rodica Pop-Busui, MD, PhD, Department of Internal Medicine, University of Michigan, Ann Arbor, MI, USA

Bruce Perkins, MD, MPH, Division of Endocrinology, University of Toronto, Toronto, Canada

Gerry Rayman, MD, Diabetes Centre, Ipswich Hospital, Ipswich, UK

James Russell, MD, Department of Neurology and Neurophysiology, University of Maryland, Baltimore, MD, USA

Søren Sindrup, MD, Department of Neurology. Odense University Hospital, Odense, Denmark

Gordon Smith, MD, Department of Neurology, University of Utah, Salt Lake City, UT, USA

Vincenza Spallone, MD, PhD, Department of Internal Medicine, University of Tor Vergata, Rome, Italy

Martin Stevens, MD, Department of Medicine, University of Birmingham, Birmingham, UK

Solomon Tesfaye, MD, Diabetes Research Unit, Sheffield Teaching Hospitals, Sheffield, UK

Paul Valensi, MD, Service d'EndocrinologieDiabétologie-Nutrition, Hôpital Jean Verdier, Bondy, France

Tamás Várkonyi, MD, PhD, First Department of Medicine, University of Szeged, Szeged, Hungary

Aristides Veves, MD, Beth Israel Deaconess Medical Center, Harvard Medical School, Boston, MA, USA

Loretta Vileikyte, MD, PhD, Department of Medicine, University of Manchester, Manchester, UK

Aaron Vinik, MD, PhD, Strelitz Diabetes Research Institutes, Eastern Virginia Medical School, Norfolk, VA, USA

Dan Ziegler, MD, Institute for Clinical Diabetology, German Diabetes Center at the Heinrich Heine University, Leibniz Center for Diabetes Research, Leibniz, Germany; Department of Metabolic Diseases, University Hospital, Düsseldorf, Germany

Doug Zochodne, MD, Department of Clinical Neuroscience, University of Calgary, AB, Canada

NIDDK observer - Teresa Jones, MD, NIDDK, Bethesda, MD, USA 


\section{References}

1. Horowitz M, Maddox AF, Wishart JM, Harding PE, Chatterton BE, Shearman DJ. Relationships between oesophageal transit and solid and liquid gastric emptying in diabetes mellitus. Eur $J$ Nucl Med 1991; 18(4): 229-234.

2. Parkman HP, Hasler WL, Fisher RS. American Gastroenterological Association technical review on the diagnosis and treatment of gastroparesis. Gastroenterology 2004; 127(5): 1592-1622.

3. Bharucha AE, Camelleri M, Forstrom LA, Zinsmeister A. Relationship between clinical features and gastric emptying disturbance in diabetes mellitus. Clin Endocrinol 2009; 70: 415-420.

4. Bytzer P, Talley NJ, Leemon M, Young LJ, Jones MP, Horowitz M. Prevalence of gastrointestinal symptoms associated with diabetes mellitus: a populationbased survey of 15000 adults. Arch Intern Med 2001; 161: 1989-1996.

5. Rayner CK, Samsom M, Jones KL, Horowitz M. Relationships of upper gastrointestinal motor and sensory function with glycemic control. Diabetes Care 2001; 24(2): 371-381.

6. Horowitz M, Edelbroek MA, Wishart JM, Straathof JW. Relationship between oral glucose tolerance and gastric emptying in normal healthy subjects. Diabetologia 1993; 36: 857-862.

7. Horowitz M, Jones KL, Rayner CK, Read NW. 'Gastric' hypoglycaemia: an important concept in diabetes management. Neurogastroenterol Motil 2006; 18: 405-407.

8. Phillips LK, Rayner CK, Jones KL, Horowitz M. An update on autonomic neuropathy affecting the gastrointestinal tract. Curr Diab Rep 2006; 6: 417-423.

9. Pasricha PJ, Pehlivanov ND, Gomez G, Vittal H, Lurken MS, Farrugia G. Changes in the gastric enteric nervous system and muscle: a case report on two patients with diabetic gastroparesis. BMC Gastroenterol 2008; 8: 21.

10. Khoo J, Rayner CK, Jones KL, Horowitz M. Pathophysiology and management of gastroparesis. Exp Rev Gastroenterol Hepatol 2009; 3: 167-181.

11. Schvarcz E, Palmer M, Aman J, Horowitz M, Stridsberg M, Berne C. Physiological hyperglycemia slows gastric emptying in normal subjects and patients with insulin-dependent diabetes mellitus. Gastroenterology 1997; 113: 60-66.

12. Jones KL, Russo A, Stevens JE, Wishart JM, Berry MK, Horowitz M. Predictors of delayed gastric emptying in diabetes. Diabetes Care 2001; 24: 1264-1269.

13. Camilleri M, Bharucha AE, Lorenzo $\mathrm{C}$, et al.Amerian Neurogastroenterology and Motility Society consensus statement on intraluminal measurement of gastrointestinal and colonic motility in clinical practice. Neurogastroenterol Motil 2008; 20: 1269-1282.

14. Jones KL, Berry M, Kong MF, Kwiatek MA, Samsom M, Horowitz M. Hyperglycaemia attenuates the gastrokinetic effect of erythromycin and affects the perception of postprandial hunger in normal subjects. Diabetes Care 1999; 22: 339-344.

15. Sturm A, Holtmann G, Goebell H, Gerken C. Prokentics in patients with gastroparesis: a systematic analysis. Digestion 1999; 60: 422-427

16. McCallum RW, Snape W, Brady F, Wa J, Parkman HP, Nowak T. Gastric electrical stimulation with Enterra therapy improves symptoms from diabetic gastroparesis in a prospective study. Clin Gastroenterol Hepatol 2010; 8. 947-954.

17. Samson M, Verhagen M. Intestinal function. In Gastrointestinal Function in Diabetes Mellitus, Horowitz M, Samsom M (eds). Chichester: John Wiley and Sons: 2004; 177-217.

18. Ma J, Stevens JE, Cukier K, et al. Effects of a protein preload on gastric emptying, glycaemia and gut hormones after a carbohydrate meal in diet controlled type 2 diabetes. Diabetes Care 2009; 32: 1600-1602.

19. Linnebjerg H, Park S, Kothare PA, et al. Effect of exenatide on gastric emptying and relationship to postprandial glycemia in type 2 diabetes. Regul Pept 2008; 151: 123-129.

20. Sun P, Cameron A, Seftel A, Shabsigh R, Niederberger C, Guay A. Erectile dysfunction - an observable marker of diabetes mellitus? A large national epidemiological study. $J$ Urol 2006; 176: 1081-1085.

21. Nicolosi A, Glasser DB, Brock G, Laumann E, Gingell C. Diabetes and sexual function in older adults: results of an international survey. $\mathrm{Br} J$ Diabetes Vasc Dis 2002; 2: 336-339.

22. Tesfaye S, Stevens LK, Stephenson JM, et al. Prevalence of diabetic peripheral neuropathy and its relation to glycaemic control and potential risk factors: the EURODIAB IDDM Complications Study. Diabetologia 1996; 39: 1377-1384.

23. Penson DF, Wessells H, Cleary P, Rutledge BN, Diabetes Control and Complications Trial/Epidemiology of Diabetes Interventions and Complications Research Group. Sexual dysfunction and symptom impact in men with long-standing type 1 diabetes in the DCCT/EDIC cohort. J Sex Med 2009; 6: 1969-1978.

24. Johannes CB, Araujo AB, Feldman HA Derby CA, Kleinman KP, McKinlay JB.
Incidence of erectile dysfunction in men 40 to 69 years old: longitudinal results from the Massachusetts male aging study. J Urol 2000; 163: 460-463.

25. Fedele D, Coscelli C, Santeusanio F, et al. Erectile dysfunction in diabetic subjects in Italy. Diabetes Care 1998; 21: 1973-1977.

26. Inman BA, Sauver JL, Jacobson DJ, et al. A population-based, longitudinal study of erectile dysfunction and future coronary artery disease. Mayo Clin Proc 2009; 84: 108-113.

27. Araujo AB, Travison TG, Ganz P, et al. Erectile dysfunction and mortality. $J$ Sex Med 2009; 6: 2445-2454.

28. Penson DF, Latini DM, Lubeck DP, Wallace KL, Henning JM, Lue TF. Do impotent men with diabetes have more severe erectile dysfunction and worse quality of life than the general population of impotent patients? Results from the Exploratory Comprehensive Evaluation of Erectile Dysfunction (ExCEED) database. Diabetes Care 2003; 26: 1093-1099.

29. De Berardis G, Pellegrini F, Franciosi M, et al. QuED (Quality of Care and Outcomes in Type 2 Diabetes) Study Group Longitudinal assessment of quality of life in patients with type 2 diabetes and self-reported erectile dysfunction. Diabetes Care 2005; 28: 2637-2643.

30. Várkonyi T, Kempler P. Erectile dysfunction. In Diabetes in Old Age (3rd edn), Sinclair A (ed). John Wiley \& Sons: 2009; 149-164.

31. Kostis JB, Jackson G, Rosen R, et al. Sexual dysfunction and cardiac risk (the Second Princeton Consensus Conference). Am J Cardiol 2005; 96: 313-321.

32. Hatzimouratidis K, Amar E, Eardley I, et al. Guidelines on male sexual dysfunction: erectile dysfunction and premature ejaculation. Eur Urol 2010; 57: 804-814.

33. Ziegler D, Merfort F, van Ahlen H, Yassin A, Reblin T, Neureither M. Efficacy and safety of flexible-dose vardenafil in men with type 1 diabetes and erectile dysfunction. J Sex Med 2006; 3: 883-891.

34. Hatzichristou D, Gambla M, RubioAurioles E, et al. Efficacy of tadalafil once daily in men with diabetes mellitus and erectile dysfunction. Diabet Med 2008; 25: 138-146.

35. Qaseem A, Snow V, Denberg TD, et al. Hormonal testing and pharmacologic treatment of erectile dysfunction: a clinical practice guideline from the American College of Physicians. Clinical Efficacy Assessment Subcommittee of the American College of Physicians. Ann Intern Med 2009; 151: 639-649.

36. Hill SR, Fayyad AM, Jones GR. Diabetes mellitus and female lower urinary tract 
symptoms: a review. Neurourol Urodyn 2008; 27: 362-367.

37. Esteghamati A, Rashidi A, Nikfallah A, Yousefizadeh A. The association between urodynamic findings and microvascular complications in patients with long-term type 2 diabetes but without voiding symptoms. Diabetes Res Clin Pract 2007; 78(1): 42-50. [E-pub 19 March 2007]

38. Jackson RA, Vittinghoff E, Kanaya AM, et al. Urinary incontinence in elderly women: finding from the Health, Aging, and Body Composition Study. Obstet Gynecol 2004; 104: 301-307.

39. Michel MC, Mehlburger L, Schumacher H, Bressel HU, Goepel M. Effect of diabetes on lower urinary tract symptoms in patients with benign prostatic hyperplasia. J Urol 2000; 163: 1725-1729.

40. Van Den Eeden SK, Sarma AV, Rutledge $\mathrm{BN}$, et al. Effect of intensive glycemic control and diabetes complications on lower urinary tract symptoms in men with type 1 diabetes: Diabetes Control and Complications Trial/Epidemiology of Diabetes Interventions and Complications (DCCT/EDIC) study. Diabetes Care 2009; 32: 664-670.

41. Sarma AV, Kanaya A, Nyberg LM, et al. Risk factors for urinary incontinence among women with type 1 diabetes: findings from the epidemiology of diabetes interventions and complications study. Urology 2009; 73(6): 1203-1209.

42. Yoshimura N, Chancellor MB, Andersson KE, Christ GJ. Recent advances in understanding the biology of DMassociated bladder complications and novel therapy. BJU Int 2005; 95: 733-738.

43. Hunter KF, Moore KN. DM-associated bladder dysfunction in the older adult (CE). Geriatr Nurs (New York, NY) 2003; 24: 138-145.

44. Lee WC, Wu HP, Tai TY, Liu SP, Chen J, Yu HJ. Effects of diabetes on female voiding behavior. $J$ Urol 2004; 172: 989-992.

45. Kaplan SA, Te AE, Blaivas JG. Urodynamic findings in patients with diabetic cystopathy. J Urol 1995; 153: 342-344.

46. Szabo L, Barkai L, Lombay B. Urinary flow disturbance as an early sign of autonomic neuropathy in diabetic children and adolescents. Neurourol Urodyn 2007; 26: 218-221.

47. Rapidi CA, Karandreas N, Katsifotis C, Benroubi M, Petropoulou K, Theodorou C. A combined urodynamic and electrophysiological study of diabetic cystopathy. Neurourol Urodyn 2006; 25: 32-38.

48. Wyndaele JJ, Kovindha A, Madersbacher H, Raddziszewski P, Ruffion A, Schurch B. Neurologic urinary and faecal incontinence. Diabetes Mellitus (916-917). In Incontinence (4th International Consultation on Incontinence) (4th edn), vol 1, Editions 21. Health Publication: Paris, 2009; 1870 p. ISBN 0-9546956-8-2.

49. Tentolouris N, Marinou K, Kokotis $P$, Karanti A, Diakoumopoulou E, Katsilambros N. Sudomotor dysfunction is associated with foot ulceration in diabetes. Diabet Med 2009; 26: 302-305.

50. England JD, Gronseth GS, Franklin G, et al. Practice parameter: evaluation of distal symmetric polyneuropathy: role of autonomic testing, nerve biopsy, and skin biopsy (an evidence-based review). Report of the American Academy of Neurology, American Association of Neuromuscular and Electrodiagnostic Medicine, and American Academy of Physical Medicine and Rehabilitation. Neurology 2009; 72: 177-184.

51. Illigens B, Gibbons C. Sweat testing to evaluate autonomic function. Clin Auton Res 2009; 19: 79-87.

52. Low VA, Sandroni P, Fealey RD, Low PA. Detection of small-fiber neuropathy by sudomotor testing. Muscle Nerve 2006; 34: 57-61.
53. Schnell O, Müller M, Standl E. Early detection of changes in the feet of diabetic patients with indicator test Neuropad. Diabetes Metab Heart 2008; 17: 203-210.

54. Quattrini C, Jeziorska M, Tavakoli M, Begum P, Boulton AJM, Malik RA. The Neuropad test: a visual indicator test for human diabetic neuropathy. Diabetologia 2008; 51: 1046-1050.

55. Low PA, Vernino S, Suarez G. Autonomic dysfunction in peripheral nerve disease. Muscle Nerve 2003; 27: 646-661.

56. Al-Moallem MA, Zaidan RM, Alkali NH. The sympathetic skin response in diabetic neuropathy and its relationship to autonomic symptoms. Saudi Med $J$ 2008; 29: 568-572.

57. Turner J, Belch JJF, Khan F. Current concepts in assessment of microvascular endothelial function using laser doppler imaging and iontophoresis. Trends Cardiovasc Med 2008; 18: 109-116.

58. Gibbons CH, Illigens BMW, Centi J, Freeman R. QDIRT: Quantitative direct and indirect test of sudomotor function. Neurology 2008; 70: 2299-2304.

59. Chao CY, Cheing CL. Microvascular dysfunction in diabetic foot disease and ulceration. Diabetes Metab Res Rev 2009; 25: 604-614.

60. Quattrini C, Harris ND, Malik RA, Tesfaye S. Impaired skin microvascular reactivity in painful diabetic neuropathy. Diabetes Care 2007; 30: 655-659.

61. Hilz MJ, Hecht MJ, Berghoff M, Singer W, Neundoerfer B. Abnormal vasoreaction to arousal stimuli-an early sign of diabetic sympathetic neuropathy demonstrated by laser Doppler flowmetry. J Clin Neurophysiol 2000; 17: 419-425.

62. Kim SW, Kim SC, Nam KC, Kang ES, Im JJ, Kim DW. A new method of screening for diabetic neuropathy using laser Doppler and photoplethysmography. Med Biol Eng Comput 2008; 46: 61-67. 